\title{
Horse shoe kidney with uretero- pelvic junction obstruction: insights in embryology and management
}

\begin{abstract}
Horseshoe kidney is the commonest fusion anomaly of the genitourinary tract with a prevalence of 1 in 400-800 live births. It is characterized by renal malrotation, variable blood supply, high insertion of the ureter, and a propensity to form an uretero-pelvic junction (UPJ) obstruction in up to one-third of cases. The patients present with abdominal pain, recurrent urinary tract infections and calculi. The treatment of choice is surgery which can be done by open or laparoscopic approach. There is no consensus on management of UPJ obstruction in children till date. Minimal access surgery is rapidly gaining popularity but has steep learning curve in children unlike adults. We are hereby reporting a case of UPJ obstruction in horse shoe kidney in a female child with on insights on embryology and management. Current management strategies and review of pertinent literature has also been provided.
\end{abstract}

Keywords: horseshoe kidney, pyeloplasty, ureteropelvic junction obstruction
Volume 7 Issue 3 - 2017

\author{
Advait Prakash,' Dipti Saxena² \\ 'Department of Pediatric Surgery, Sri Aurobindo Institute of \\ medical sciences \& Post graduate Institute, India \\ ${ }^{2}$ Department of Anesthesiology, Sri Aurobindo Institute of \\ medical sciences \& Post graduate Institute, India
}

Correspondence: Advait Prakash (MS, MCh), Associate Professor, Department of Pediatric Surgery, Sri Aurobindo institute of medical sciences\& Post graduate Institute, Indore (M.P.), India, Tel 919000000000 ,

Email drprakashadvait@rediffmail.com

Received: August 22, 2017 | Published: November 15, 2017

Abbreviations: UPJ, ureteropelvic junction; UPJO, ureteropelvic junction obstruction

\section{Introduction}

Horseshoe kidney is the most common fusion anomaly of the kidney, with an incidence of approximately $0.25 \%$ of the general population. It is more frequently observed in males (M:F 2:1). ${ }^{1}$ Horseshoe kidneys are frequently associated with other congenital anomalies and ureteropelvic junction obstruction (UPJO). ${ }^{1}$ Horseshoe kidney is a renal fusion anomaly characterized by renal malrotation, variable blood supply, high insertion of the ureter, and a propensity to form ureteropelvic junction (UPJ) obstruction in up to one third of cases. ${ }^{2}$ There is still no consensus regarding modality of management of UPJO in children. Minimal access surgery is rapidly gaining popularity but has steep learning curve in children unlike adults. We are hereby reporting a case of UPJ obstruction in horse shoe kidney in a 10 year old female child along with insights in embryology and management with status of various surgical modalities in current scenario.

\section{Case report}

A 10 year old female presented with chronic vague left abdominal pain with burning micturition since last 2 years. Clinical examination and renal function tests were unremarkable. Ultrasonography (USG) showed presence of low lying kidneys close to the vertebral column along with medially pointing lowermost calyx. Anteriorly displaced upper ureter and left side PUJO were also noted (Figure 1). Similar findings were seen on renal nuclear scan along with decreased function and thin parenchyma on left side (Figure 2). Modified Anderson-Hynes pyeloplasty was performed without isthmectomy transperitoneally. The narrowed pelviureteric junction was resected and ureter distal to PUJO was spatulated (Figure 3). Redundant pelvis was excised and a dependent anastomosis was performed over a $4 \mathrm{Fr}, 20 \mathrm{~cm}$, double-J stent (D-J stent) (Figure 4). Patient recovered uneventfully and was discharged on third post-operative day. Patient presently is asymptomatic and on regular follow up for 20 months. Post-operative serial ultrasound and renal scan have shown improvement in the renal function, parenchymal thickness and resolution of hydronephrosis.

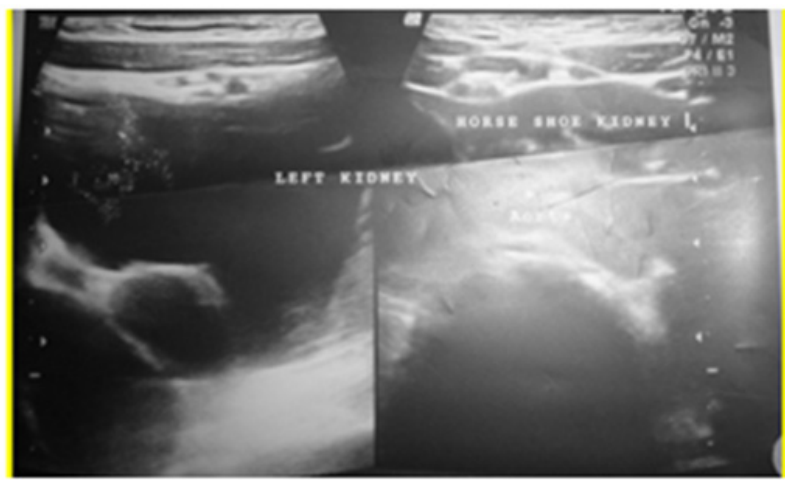

Figure I USG abdomen showing horse shoe kidney.

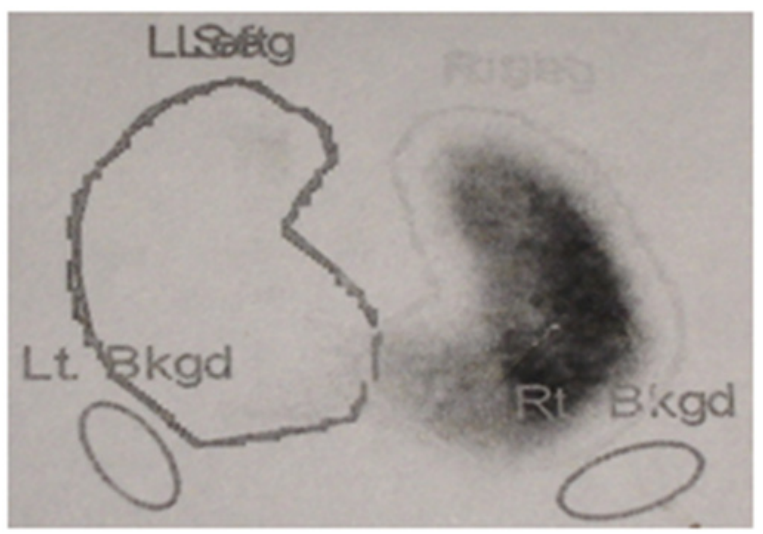

Figure 2 Nuclear renal scan showing horse shoe kidney.

\section{Discussion}

Horse shoe kidney is one of the most common fusion anomaly of kidney development found in approximately $0.25 \%$ of the general population. $^{3}$ It results from fusion of metanephric buds between 4 and 8 weeks of embryogenesis which hampers their cephalic migration and normal rotation. ${ }^{3}$ The renal pelvis and ureters in horseshoe kidney are anteriorly placed, with the ureters lying 
anterior to the isthmus with a tendency to kink, often resulting in hydronephrosis. ${ }^{4}$ UPJ obstruction is postulated to develop secondary to congenital stricture, high ureteral insertion, an abnormal ureteral course over the isthmus, crossing vessels supplying the isthmus, or abnormal motility of the UPJ segment. ${ }^{5}$ Hydronephrosis from PUJO occurs in about $14 \%$ to $35 \%$ of cases of horse shoe kidney. ${ }^{6,7}$ The anomaly is generally detected incidentally. If symptomatic it is related to hydronephrosis, infection, or calculus formation. The most common symptom is vague abdominal pain radiating to the lower lumbar region The Rovsing sign which includes abdominal pain, nausea and vomiting on hyper-extension of the spine has not been observed commonly. ${ }^{1}$ Clinical findings are those of infection, calculi, obstruction or tumor due to anomalous position of pelvis, and ureters. As the most common complication of the horseshoe kidney necessitating surgical intervention, urolithiasis has an incidence of $20-60 \%$, and UPJ obstruction occurs at an incidence of $15-33 \%{ }^{8}$ The primary technical challenges of pyeloplasty in this population relate to aberrant lower pole vessels, unfamiliar caudal position of the kidney, and the renal isthmus. ${ }^{5}$. Despite these anatomical challenges to optimize success ,anatomic complexities can be anticipated with appropriate preoperative imaging. ${ }^{9}$ On imaging studies, the classic findings include: low lying kidneys close to the vertebral column, collecting system directly posterior to each renal pelvis, lowermost calyx pointing medially and the high insertion of the ureter into the pelvis. ${ }^{1}$ Ultrasound and renal dynamic scan are usually sufficient for the diagnosis, management and follow-up of patients of horseshoe kidney with PUJO. ${ }^{3}$ Ultrasound is very accurate and sensitive in these patients, which reduces the need for cross-sectional imaging in most cases. ${ }^{3} \mathrm{~A}$ CT angiogram can accurately delineate the vasculature and collecting system. ${ }^{5}$ Magnetic resonance urography (MRU) can also be utilized for simultaneous evaluation of renal function and anatomy. 5,9

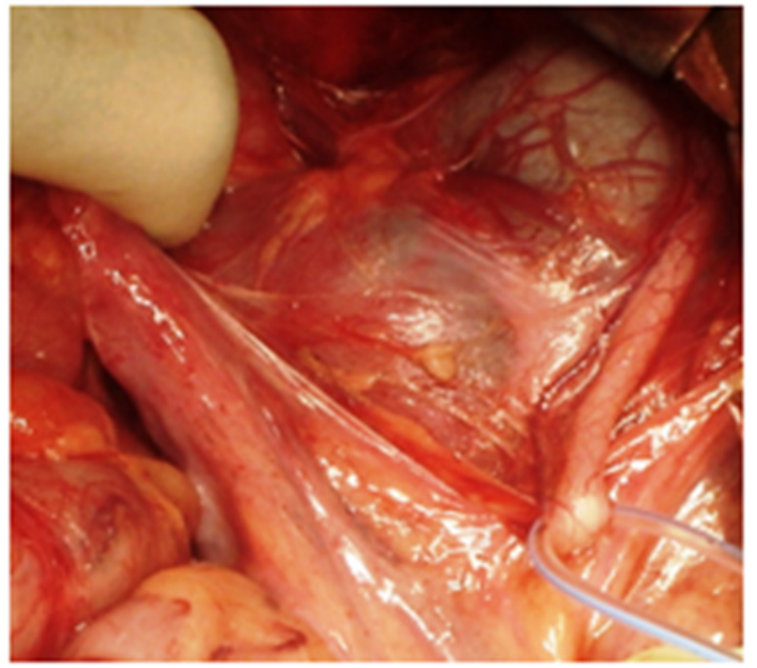

Figure 3 Intra-opeartive photograph showing horse shoe kidney with isthmus and slinged ureter.

Treatment is surgical either by open flank approach (extraperitoneal for unilateral and trans-peritoneal for bilateral involvement) or by laparoscopy or robotic surgery. Surgical management in the form of excision of stenotic ureteropelvic segments, trimming of a redundant pelvis, transposition of any aberrant vessels, and ureteropelvic anastomosis are the basic principles of management. ${ }^{3}$ Horseshoe UPJ obstruction caused by an intrinsic stenosis, high ureteral insertion, or abnormal course of the ureter across the isthmus can be addressed appropriately by dismembered pyeloplasty. ${ }^{5}$ Other studies have also suggested that the obstruction is not due to pressure at the place where the ureter crosses the kidney. They also concluded that a dismembering pyeloplasty is the procedure of choice for treating PUJO in horse shoe kidneys. ${ }^{10}$ UPJ in horse shoe kidney can be reached by trans-peritoneal approach by a lower midline or transverse incision or by the retroperitoneal approach via a flank incision. An excellent exposure of the UPJ in horse shoe kidneys has been observed by both the approaches. ${ }^{3,10}$ The resolution of hydronephrosis and drainage after Anderson-Hynes pyeloplasty in horseshoe kidneys is comparable to that in normally situated hydronephrotic kidneys. ${ }^{10}$ On the other hand, success rate of the Foley Y-V plasty reaches nearly $80 \%{ }^{7}$

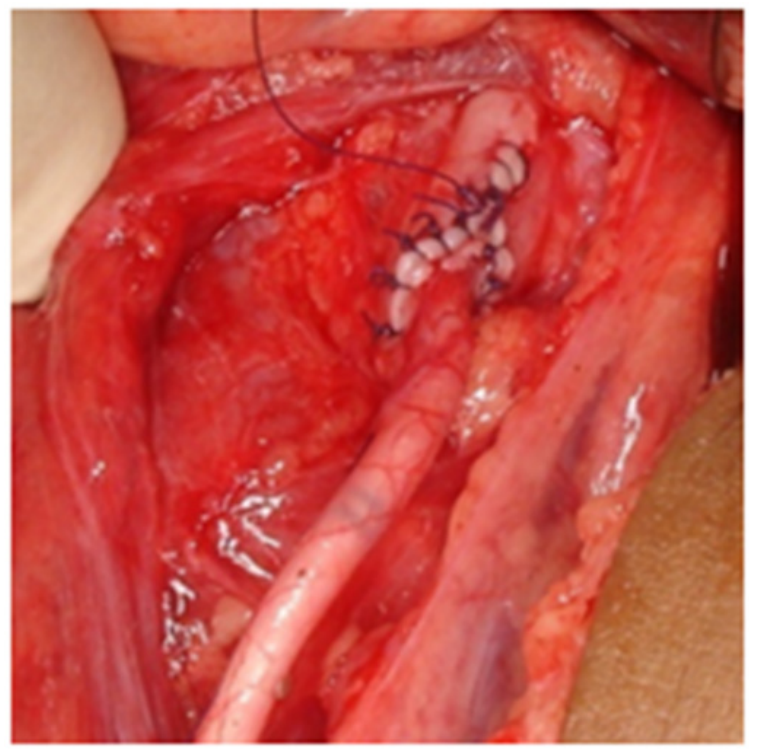

Figure 4 Completed pyeloplasty in HSK.

A crossing vessel causing obstruction in isolation can theoretically be treated with either a vascular hitch or dismembered pyeloplasty. Prior studies have suggested the best candidates for a Hellstrom repair are those with a crossing vessel and a normal caliber ureter with good peristalsis across the UPJ. ${ }^{11}$ Division of the isthmus has been a topic of debate during pyeloplasty in such patients. Division of the isthmus is not mandatory to ensure adequate drainage. Isthmotomy also contains risk of partial ischemia due to its variable blood supply. Modified Anderson-Hynes pyeloplasty via a flank incision without additional division of the isthmus is also a highly effective and safe procedure for treating PUJO in horseshoe kidney. ${ }^{10}$ Due to the rarity of horseshoe kidney, large case series will take time to occur, which limits our ability to interpret the outcomes and/or advantages of various techniques.

The management of UPJO has evolved dramatically over last decade and endoscopic and laparoscopic procedures are replacing open pyeloplasty for primary UPJO. Laparoscopic pyeloplasty in children is a technically demanding procedure requiring advanced technical skills to perform this complex reconstructive procedure. Hence it is being performed in a few specialized centers. Peri-operative outcomes mirrored those for laparoscopic and robotic pyeloplasty in orthotopic kidney. It is believed that proficiency obtained during laparoscopic repair in orthotopic kidneys reasonably translates into competency in horseshoe kidney pyeloplasty. ${ }^{9}$ The choice of approach may be influenced by surgeon's experience, patient expectations and resource allocation, but both laparoscopy and robotic approaches appear to be safe and technically feasible in adult population. This is not duplicated in pediatric population. Among pediatric population the learning curve of laparoscopic pyeloplasty is very steep owing to the space constraint, associated anomalies and anesthetic challenges. 
Endopyelotomy which has been suggested as the surgical procedure of choice for release of PUJO in HSK in adults, is not so beneficial in treating children. ${ }^{12}$

There should be a high index of suspicion for UPJ obstruction in a patient presenting with horseshoe kidney with symptoms. Its management requires multi-modal approach with the judicious use of endoscopic and open surgical intervention. Minimally invasive approaches have become gold standard in adults. In pediatric age group also they are rapidly gaining popularity but require high level of expertise and although they behold good future, these cannot be recommended as primary management at present. Correction of UPJ obstruction is recommended to treat the symptoms. AndersonHynes pyeloplasty by a flank incision without additional division of the Isthmus of the kidney is a highly effective and safe procedure for treating UPJ obstruction in horse shoe kidney in children.

\section{Acknowledgments}

None.

\section{Conflicts of interest}

The authors declare no conflicts of interest.

\section{References}

1. Shapiro E, Bauer SB, Chow JS. Anomalies of the upper urinary tract. In:Wein AJ, et al. editors. Campbell-Walsh Urology. 10th edn, Elsevier Saunders, Philadelphia, USA. 2012. pp. 3123-3161.

2. Lallas $\mathrm{CD}$, Pak RW, Pagnani $\mathrm{C}$, et al. The minimally invasive management of ureteropelvic junction obstruction in horseshoe kidneys. World J Urol. 2011;29(1):91-95.
3. Panda SS, Bajpai M, Jana M, et al. Anderson-Hynes pyeloplasty with isthmotomy and lateropexy in horseshoe kidneys with pelviureteric junction obstruction in children. Indian J Urol. 2014;30(2):161-163.

4. Kehagias DT, Gouliamos AD, Vlahos LJ. Horseshoe kidney associated with anomalous inferior vena cava. Eur Radiol. 1999;9(5):935-936.

5. Yohannes P, Smith AD. The endourological management of complications associated with horseshoe kidney. J Urol. 2002;168(1):58.

6. Das S, Amar AD. Ureteropelvic junction obstruction with associated renal anomalies. J Urol. 1984;131(5):872-874.

7. Pitts WR Jr, Muecke EC. Horseshoe kidneys:A 40-year experience. $J$ Urol. 1975;113(6):743-746.

8. Singhania PP, Raut NR, Shringarpure SS, et al. Horseshoe Kidney with Bilateral Ureteropelvic Junction Obstruction with Multiple Renal Calculi: A Case Report. Int J Sci Stud. 2015;3(6):233-235.

9. Faddegon S, Granberg C, Tan YK, et al. Minimally invasive pyeloplasty in horseshoe kidneys with ureteropelvic junction obstruction:A case series. Int Braz J Urol. 2013;39(2):195-202.

10. Schuster T, Dietz HG, Schütz S. Anderson-Hynes pyeloplasty in horseshoe kidney in children:Is it effective without symphysiotomy? Pediatr Surg Int. 1999;5(3-4):230-233.

11. Gundeti MS, Reynolds WS, Duffy PG, et al. Further experience with the vascular hitch (laparoscopic transposition of lower pole crossing vessels): an alternate treatment for pediatric ureterovascular ureteropelvic junction obstruction. J Urol. 2008;180(4 Suppl):1832-1836.

12. Nakamura K, Baba S, Tazaki H. Endopyelotomy in horseshoe kidneys. J Endourol. 1994;8(3):203-206. 